\title{
Non-Invasive Evaluation and Differential Diagnosis for Pediatric Leukoderma in a Single Institute
}

\author{
Madoka Takafuji*, Atsushi Tanemura*, Yuma Hanaoka, Noriko Arase, Eiji Kiyohara, \\ Ichiro Katayama, Manabu Fujimoto
}

Department of Dermatology, Osaka University Graduate School of Medicine, Suita, Japan

Email: madoka_n_n_0822@yahoo.co.jp, tanemura@derma.med.osaka-u.ac.jp

How to cite this paper: Takafuji, M., Tanemura, A., Hanaoka, Y., Arase, N., Kiyohara, E., Katayama, I. and Fujimoto, M. (2019) Non-Invasive Evaluation and Differential Diagnosis for Pediatric Leukoderma in a Single Institute. Journal of Cosmetics, Dermatological Sciences and Applications, 9, 313-320.

https://doi.org/10.4236/jcdsa.2019.94028

Received: October 24, 2019

Accepted: November 26, 2019

Published: November 29, 2019

Copyright $\odot 2019$ by author(s) and Scientific Research Publishing Inc. This work is licensed under the Creative Commons Attribution International License (CC BY 4.0).

http://creativecommons.org/licenses/by/4.0/

\begin{abstract}
Background: Although vitiligo shows a similar clinical picture to other disorders in which patients exhibit depigmented spots, there are few reports on the differentiation of these conditions from each other. Purpose: We aimed to investigate the differences and similarities between vitiligo and nevus depigmentosus in children based on the clinical course and characteristics. Materials and Methods: We conducted an examination of the disease type, treatment, progress, and differentiation of 85 cases of vitiligo and nevus depigmentosus that developed before 15 years of age among patients who visited the Osaka University Department of Dermatology with a chief complaint of white patches from January 2010 to March 2017. Results: The median age at the onset of symptoms of 79 patients with vitiligo was 4.5 years and while that of 6 patients with nevus depigmentosus was 0.8 years, indicating that the onset of nevus depigmentosus occurred earlier. While vitiligo often developed in the head and neck regions, with a tendency for depigmented spots in multiple regions, nevus depigmentosus did not show this tendency in these regions. Upon performing Wood's lamp observation in 38 cases of vitiligo, 35 cases were found to be fluorescent chalky white and in 3 cases histologically diagnosed as vitiligo, no clear fluorescent color was observed. All 6 patients diagnosed with nevus depigmentosus exhibited a non-fluorescent off-white color under a Wood's lamp. Conclusions: This study indicates that non-invasive observation using a Wood's lamp is useful in the differential diagnosis of pediatric leukoderma, in addition to the subsequent clinical course, such as the presence or absence of lesional repigmentation and/or enlargement.
\end{abstract}

\section{Keywords}

Pediatric Leukoderma, Vitiligo, Nevus Depigmentosus, Non-Invasive Diagnosis, Wood's Lamp

*These authors contributed equally to this work. 


\section{Introduction}

Vitiligo is an acquired condition associated with the manifestation of spots of complete depigmentation with a round or irregularly-shaped white macule [1]. On the other hand, nevus depigmentosus is a congenital condition associated with the manifestation spots of incomplete depigmentation characterized by a single onset on the trunk and limbs, with a serrated periphery. However, it has been reported that nevus depigmentosus was detected at birth in less than $7.5 \%$ of cases, with approximately $20 \%$ of cases diagnosed after 3 years of age, which is similar to the clinical picture of vitiligo, particularly localized or segmental-type vitiligo [2]. A proper differential diagnosis of these diseases is critically important because a misdiagnosis may lead to redundant treatment and an insufficient explanation to guardians. However, there are few cumulative studies describing a differential diagnosis by non-invasive methods rather than histopathological examination [3]. A Wood's lamp emits UV at wavelengths of 320 to $400 \mathrm{~nm}$. Under Wood's lamp illumination, the borders of hypopigmented or depigmented lesions show increased sharpness and bright blue-white fluorescence due to the presence of dermal collagen and porphyrins. This results from decreased amount or absence of intervening melanin [4]. In common, complete hypopigmentation, including vitiligo, shows a chalky-white color with fluorescence while incomplete hypopigmentation, including nevus depigmentosus, exhibits an off-white color without fluorescence [5]. In this study, we attempted to diagnose pediatric vitiligo and nevus depigmentosus at an early stage through non-invasive evaluations rather than by histopathological examination.

\section{Methods}

\subsection{Patients}

We retrospectively examined 79 patients with vitiligo and 6 patients with nevus depigmentosus, in whom the onset of the condition occurred before 18 years of age and who visited the Osaka University Department of Dermatology with a chief complaint of white patches from January 2010 to March 2017. The following parameters were evaluated and compared between vitiligo and nevus depigmentosus patients: sex, age at visit, age at onset, region of onset, past medical history (only for patients who had received an inquiry or consented to blood sampling), clinical course, and therapeutic effect. The therapeutic effect was evaluated as either of the following changes: complete or partial repigmentation, no repigmentation, or the enlargement of depigmentation. This clinical study was approved by Osaka University Clinical Research Review Committee.

\subsection{Examination of Lesions by Histopathology and Wood's Lamp}

An examination by observation with a Wood's lamp (TheraBeam ${ }^{\mathrm{TM}}$ Woody, USHIO Inc., Tokyo, Japan) was performed for 38 patients and the results were collated with results obtained from the long-term observation of the clinical course. A histopathological examination was performed for 10 patients to ac- 
quire a definitive diagnosis and measure remaining melanocytes for further treatment. The remaining melanin and melanocytes were visualized by Masson-Fontana and Melan A staining, respectively. Pictures captured under a Wood's lamp were evaluated by 3 independent dermatologists to avoid subjective bias.

\section{Results}

1) Age at first visit, timing of the onset of symptoms, and sex

The details are summarized in Table 1 . The study population included 85 patients with of vitiligo or nevus depigmentosus (male, $n=37$ [43\%], female, $n=$ 48 [57\%]; age at the first visit, 0 - 15 years), among patients with depigmentation that developed before 15 years of age. Seventy-nine (93\%) of the patients were diagnosed with vitiligo (male, $\mathrm{n}=35$ [44\%]; female, $\mathrm{n}=44$ [56\%]; age at onset of symptoms 0 - 15 years [median, 4.5 years]). Six patients (7\%) were diagnosed with nevus depigmentosus (male, $\mathrm{n}=2$ [33\%] female, $\mathrm{n}=4$ [67\%]; age at onset of symptoms, 0 - 3 years [median, 0.8 years]). The age at the first visit was 4.5 years for vitiligo and 3.0 years for nevus depigmentosus.

2) Site of onset

The region of onset was examined separately for the head, face, neck, upper limbs, trunk, pubic area, groin, and lower limbs. The sites of vitiligo lesions were as follows: head (5 patients), face (43 patients), neck (20 patients), upper limb (15 patients), trunk (31 patients), pubic area (6 patients), and lower limb (16 patients). Depigmented spots were often observed at multiple sites in a single case,

Table 1. Demographics of the patients enrolled in the present study.

\begin{tabular}{|c|c|c|}
\hline & VV & ND \\
\hline & $(n=79)$ & $(n=6)$ \\
\hline \multicolumn{3}{|l|}{ Gender } \\
\hline Male & 35 & 2 \\
\hline Female & 44 & 4 \\
\hline Age of the 1st visit, years (range) & $4.5(0-15)$ & $3.0(0-6)$ \\
\hline Age of disease notification, years (range) & $4.3(0-15)$ & $0.8(0-3)$ \\
\hline & atopic dermatitis: 9 & \\
\hline \multirow{3}{*}{ Comorbidity } & asthma: 5 & \\
\hline & TPO-Ab positive: 1 & none \\
\hline & FT3 high: 1 & \\
\hline \multicolumn{3}{|l|}{ Course of clinical feature } \\
\hline Complete or partial repigmentation & 58 & 0 \\
\hline No change & 8 & 6 \\
\hline Disease progression & 7 & 0 \\
\hline Unevaluable & 6 & \\
\hline
\end{tabular}

VV, Vitiligo Vulgaris; ND, Nevus Depigmentosus. 
even within the same region. The sites of nevus depigmentosus lesion were as follows: head (1 patient), neck ( 2 patients), upper limb (1 patient), trunk (2 patients), and lower limb (1 patient). Five of 6 nevus depigmentosus cases involved a single region, indicating a tendency towards a smaller number of regions in comparison to vitiligo cases.

3) Clinical and histopathological findings

Approximately $70 \%$ of vitiligo cases had depigmented spots scattered throughout the body, which made it possible to make a definitive diagnosis of vitiligo. However, it is difficult to differentiate vitiligo from nevus depigmentosus in pediatric cases in which only a solitary and tiny depigmented spot is observed. In these cases, the clinical course, such as whether the depigmented spot expands or whether repigmentation is acquired, are also important for the diagnosis. While vitiligo often changes in shape and range over the natural course [6], nevus depigmentosus is commonly resistant to any treatment [2]. Fifty-eight (73\%) of the 91 vitiligo patients experienced partial or complete pigment regeneration over the course of therapy (Table 1). In contrast, none of the patients with nevus depigmentosus exhibited repigmentation in the due course as previously reported ( 6 of 6 cases; 100\%). Among 38 patients with vitiligo who were observed with a Wood's lamp, 35 (92\%) who were diagnosed without a skin biopsy had lesions that were fluorescent chalky-white [5], 3 had non-fluorescent off-white lesions, and 1 coexisting vitiligo and nevus depigmentosus. In contrast, the lesions in all 6 cases of nevus depigmentosus exhibited an off-white appearance. A definitive diagnosis by histopathology was only required for 3 of the 38 vitiligo cases that exhibited an off-white appearance under a Wood's lamp. The following representative cases demonstrate findings in patients who underwent Wood's lamp observation and a histopathological examination.

\section{Case 1: Vitiligo}

The patient was a 13-year-old girl, with segmental vitiligo that occurred on the right thigh. The Wood's lamp excited fluorescence at the first visit, and the patient was diagnosed with segmental vitiligo (Figure 1(a) and Figure 1(b)). Treatment with topical tacrolimus and steroids every other day was initiated. We found a remarkable decrease in basal melanosis and a complete loss of melanocytes (Figure 2(a) and Figure 2(b)).

Case 2: Nevus depigmentosus

The patient was a 1-year-old boy, with nevus depigmentosus on the right forearm. There has been no change in shape since the first visit. The periphery was serrated. The Wood's lamp did not excite fluorescence, and the lesion showed an off-white appearance (Figure 1(c) and Figure 1(d)). We found a slight decrease in basal melanosis but no decrease in the number of melanocytes (Figure 2(c) and Figure 2(d)).

Case 3: Segmental vitiligo coexistent with nevus depigmentosus

The patient was a 5-year-old girl with depigmentation on her left forehead and left chest that had been noticed 3 years previously. The patient had no relevant medical history or family history, and her twin sister did not exhibit the 

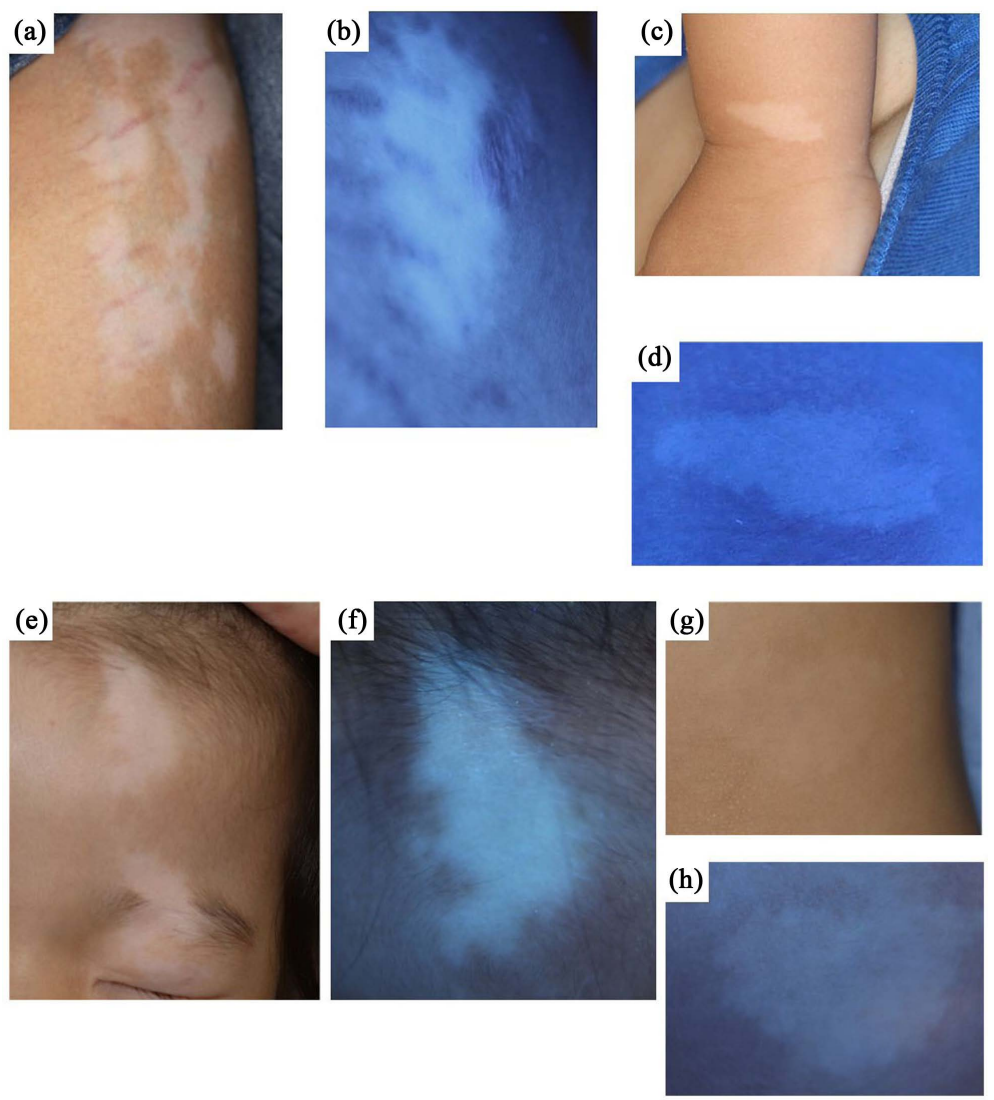

Figure 1. (a) Vitiligo lesion on the right medial thigh in case 1; (b) (a) exhibits a chalky white appearance with fluorescence under a Wood's lamp; (c) Nevus depigmentosus lesion on the right wrist in case 2; (d) (c) exhibits an off-white appearance without fluorescence under a Wood's lamp. Case 3 suffered from segmental vitiligo on the left forehead ((e) and (f) ) and nevus depigmentosus on the left flank ((g) and (h)). The different appearance under a Wood's lamp is demonstrated in (f) and (h).
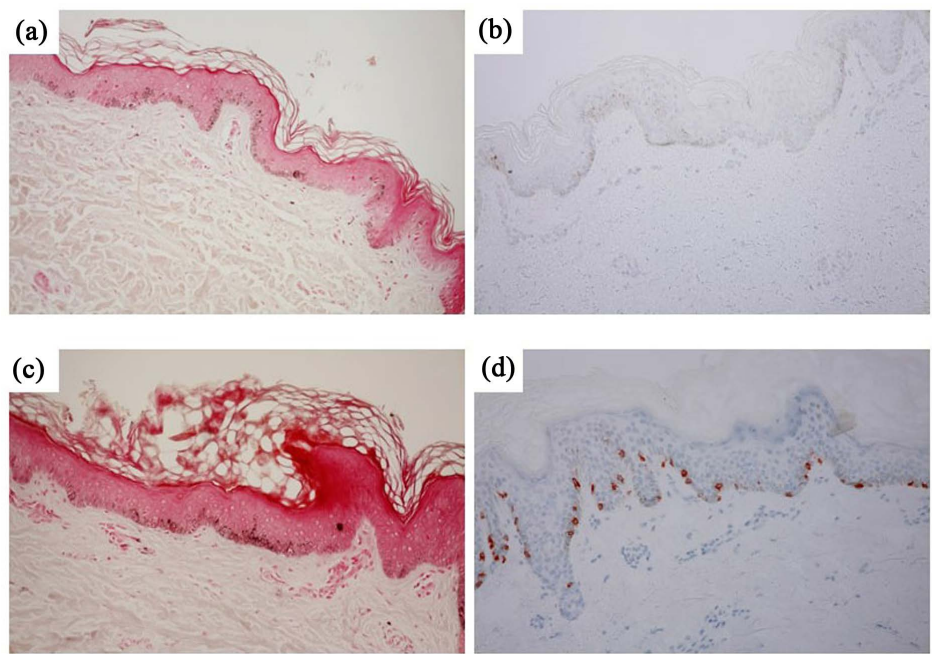

Figure 2. A remarkable decrease in basal melanosis and a complete loss of melanocytes was detected in case 1 Masson-Fontana and Melan A staining, respectively ((a), (b)). In contrast, slight decrease in basal melanosis and no reduction in melanocytes were found in case 2 by Masson-Fontana and Melan A staining, respectively ((c), (d)). 
condition. Under a Wood's lamp, her forehead exhibited a chalky-white color (Figure 1(e) and Figure 1(f)) and the depigmented spots on her chest exhibited an off-white color (Figure 1(g) and Figure 1(h)). Accordingly, she was diagnosed with coexisting vitiligo (forehead) and nevus depigmentosus (chest).

\section{Discussion}

Vitiligo is known to affect approximately $1 \%$ of the population; approximately half to one-third of cases occur in childhood [7]. Although vitiligo lesions generally show complete depigmentation, they may show incomplete and/or uneven depigmentation in the early stage [1] and it is possible for lesions to extend throughout the body. On the other hand, nevus depigmentosus clinically exhibits incomplete depigmentation with an appearance that varies, from single and irregularly shaped spots to lesions arranged in strips or with serrated edges [6]. The following diagnostic criteria for nevus depigmentosus were proposed by Coupe: 1) onset at birth or early age; 2) no change in distribution of depigmentation throughout life; 3) no change in texture of depigmented spots; and 4) no pigment enhancement around the depigmented area [8]. The most common region of nevus depigmentosus is the trunk of the body, and on histopathological examination, no decrease in the number of melanocytes is observed. Among cases in which a biopsy was possible, the results of Masson-Fontana and Melan A staining confirmed that there was no decrease in melanocytes in the hypopigmented area. However, among cases in which a biopsy was refused, this study showed that it was useful to confirm images that did not fluoresce under a Wood's lamp.

The advantage of making a definitive diagnosis of vitiligo is that an early diagnosis enables treatment to be initiated in childhood. In patients who receive treatment, regeneration may be achieved in response to treatment or the condition may progress despite treatment. A previous study revealed that depigmentation of nevus depigmentosus was noticed before 1 year of age in 38 patients (56.7\%) and before 3 years of age in 62 patients (92.5\%) [2]. Although a skin biopsy is the best way to make a definitive diagnosis, it should be avoided as much as possible due to its invasiveness.

Whether the depigmentation of a spot is complete or incomplete has been evaluated by dermoscopy in recent years, and this has contributed to the diagnosis of vitiligo [9]. However, dermoscopy is associated with a disadvantage in that it is based on local observation within very narrow range. A Wood's lamp makes up for these shortcomings and allows the observer to detect the presence or absence of melanin over a wider angle and in more detail. With the development of LED optical technology, Wood's lamps can emit UVA, especially at wavelengths of 365 to $385 \mathrm{~nm}$. Because normal skin and depigmented spots can be distinguished more clearly in comparison to conventional UV light from fluorescent lamps, the potential exists for various methods to be applied going forward, including the evaluation of melanogenesis activity, which cannot be evaluated by 
biopsy alone, by observing the type of remaining pigmentation [10]. The proper differentiation of depigmented spots that occurred in childhood is often required, with many conditions, such as leukoderma and Ito vitiligo, developing from early childhood in association with piebaldism or tuberous sclerosis [11]. In this way, the Wood's lamp enables the minimally invasive diagnosis of pediatric leukoderma as well as the accurate evaluation of the presence and patterns of repigmentation. The present study was associated with several limitations, including the relatively small number of pediatric patients and the difference in the number of patients in the vitiligo and nevus depigmentosus groups. The increase in pediatric patients who undergo Wood's lamp observation is further required for proper assessment.

\section{Conclusion}

We evaluated pediatric leukoderma lesions based upon the clinical course and features observed under a Wood's lamp. This study indicates that non-invasive observation using a Wood's lamp is useful for the differential diagnosis of pediatric leukoderma, in addition to following the clinical course to detect features such as the presence or absence of lesional repigmentation and/or enlargement.

\section{Conflicts of Interest}

The authors declare no conflicts of interest in association with the present study.

\section{References}

[1] Taieb, A. (2000) Intrinsic and Extrinsic Pathomechanisms in Vitiligo. Pigment Cell Research, 13, 41-47. https://doi.org/10.1034/j.1600-0749.13.s8.9.x

[2] Lee, H.S., Chun, Y.S. and Hann, S.K. (1999) Nevus Depigmentosus: Clinical Features and Histopathologic Characteristics in 67 Patients. Journal of the American Academy of Dermatology, 40, 21-26. https://doi.org/10.1016/S0190-9622(99)70524-4

[3] Xu, A.E., Huang, B., Li, Y.W., Wang, P. and Shen, H. (2008) Clinical, Histopathological and Ultrastructural Characteristics of Naevus Depigmentosus. Clin Exp Dermatol; 33, 400-405. https://doi.org/10.1111/j.1365-2230.2008.02714.x

[4] Asawanonda, P. and Taylor, C.R. (1999) Wood's Light in Dermatology. International Journal of Dermatology, 38, 801-807. https://doi.org/10.1046/j.1365-4362.1999.00794.x

[5] Taieb, A. and Picardo, M. (2010) Vitiligo. 2010th Edition, Springer, New York. https://doi.org/10.1007/978-3-540-69361-1

[6] Mu, E.W., Cohen, B.E. and Orlow, S.J. (2015) Early-Onset Childhood Vitiligo Is Associated with a More Extensive and Progressive Course. Journal of the American Academy of Dermatology, 73, 467-470. https://doi.org/10.1016/j.jaad.2015.05.038

[7] Silverberg, N.B. (2015) The Epidemiology of Vitilligo. Current Dermatology Reports, 4, 36-43. https://doi.org/10.1007/s13671-014-0098-6

[8] Coupe, R.L. (1976) Unilateral Systematized Achromic Naevus. Dermatologica, 134, 19-35. https://doi.org/10.1159/000254235

[9] Al-Refu, K. (2018) Dermoscopy Is a New Diagnostic Tool in Diagnosis of Common 
Hypopigmented Macular Disease: A Descriptive Study. Dermatology Reports, 11, 1-7. https://doi.org/10.4081/dr.2018.7916

[10] Bae, J.M. and Han, T.Y. (2017) A Grayscale Photograph with High Dynamic Range Taken under a Wood's Lamp for Better Recognition of Vitiligo Lesions. Journal of the American Academy of Dermatology, 76, 89-90.

https://doi.org/10.1016/j.jaad.2016.11.011

[11] Ezzedine, K., Lim, H.W., Suzuki, T., et al. (2012) Revised Classification/Nomenclature of Vitiligo and Related Issues: The Vitiligo Global Issues Consensus Conference. Pigment Cell \& Melanoma Research, 25, E1-E13.

https://doi.org/10.1111/j.1755-148X.2012.00997.x 